\title{
El Legado de Johannes Diderik van der Waals y su Conferencia Nobel
}

José O. Valderrama ${ }^{(1,2)}$

(1) Univ. de La Serena, Fac. de Ingeniería, Dpto. de Ing. Mecánica., Casilla 554, La Serena-Chile

(2) Centro de Información Tecnológica (CIT), Casilla 724, La Serena-Chile

(e-mail: jvalderr@userena.cl)

*La Conferencia Nobel es traducida con la debida autorización de la Fundación Nobel, Estocolmo-Suecia

\section{Resumen}

El autor presenta la conferencia que dictó Johannes Diderik van der Waals cuando recibió el premio Nobel de física el año 1910. Este trabajo es una contribución a las celebraciones que se realizan alrededor del mundo para conmemorar los cien años desde que van der Waals recibiera el máximo galardón. La conferencia resume en forma simple pero detallada algunos de sus principales logros; en particular la ecuación de estado, el principio de estados correspondientes y la teoría de mezclas. Estas pioneras ideas de van der Waals han influenciado efectivamente varias áreas de la ciencia, pero en particular estos tres conceptos, y que fueron los que motivaron su merecido premio Nobel. Este artículo concluye que aún después de 100 años del Nobel y más de 125 de la Tesis doctoral, y a pesar de muchos otros extraordinarios logros en la física y en la termodinámica, los conceptos de van der Waals siguen más vigentes que nunca.

Palabras clave: van der Waals, ecuaciones de estado, premio Nobel, conferencia Nobel

\section{The Legacy of Johannes Diderik van der Waals and his Nobel Lecture}

\begin{abstract}
The author presents the conference that van der Waals delivered when he received the Nobel Prize for Physics in 1910. This work is a contribution to the several commemorating activities that are being organized around the world to celebrate the 100 years since van der Waals was awarded the maximum prize. The conference summarizes some of the main achievements and in particular the equation of state, the principle of corresponding states and the theory of mixtures. These pioneering ideas of van der Waals have influenced several areas of physics and thermodynamics but in particular these three concepts, which motivated his merited Nobel Prize. The paper concludes that even after a hundred years since the Nobel and more than 125 years since the doctoral thesis, and despite of many other extraordinary advances in physics and thermodynamics, the concepts of van der Waals continue more valid than ever.
\end{abstract}

Keywords: van der Waals, equations of state, Nobel Prize, Nobel lecture 


\section{INTRODUCCIÓN}

En diciembre de este año 2010, se cumplen cien años desde que Johannes Diderick van der Waals recibiera el Premio Nobel de Física, en reconocimiento a sus pioneros estudios sobre la física de líquidos y gases (Nobel.org., 2010a). La ocasión es entonces propicia para reflexionar sobre la obra y legado de este notable físico holandés. En particular se presenta la extraordinaria Conferencia Nobel que presentó cuando recibió el máximo galardón, el 12 de diciembre del año 1910 (van der Waals, 1910). A partir de su trabajo de Tesis (cuya portadilla se muestra en la Fig. 1), van der Waals (1873) presentó unos años más tarde el concepto de estados correspondientes y su teoría de mezclas. Son precisamente estas y otras consecuencias de su trabajo de Tesis las que lo llevaron a perseverar en el tema y que lo condujeron a su merecido premio Nobel. Pero a pesar de este reconocimiento, para van der Waals no fue fácil imponer sus revolucionarias ideas. Creencias científicas como que una sustancia líquida era algo diferente que su vapor, que habían algunos gases que no podían ser convertidos en líquidos, que las moléculas en la fase gas no ocupaban espacio y que además no interactuaban entre ellas, fueron conceptos no fácilmente aceptados por algunos connotados científicos de la época. En su Conferencia Nobel, van der Waals se encargaría de encararlos y sin falsa modestia dejar establecido que su obra daría que hablar. "Muchos de aquellos que se oponían han sido finalmente derrotados y mi teoría puede haber sido un factor determinante. Y precisamente esto, creo yo, representa un avance".

Parte de las varias contribuciones de van der Waals han tenido tremendas repercusiones en el estudio de la física de fluidos y áreas afines. Su ecuación fue el primer modelo que incorporaba la transición líquido gas y los fenómenos críticos. Sus contribuciones en el tratamiento de mezclas binarias y el principio de estados correspondientes son descubrimientos trascendentales que justifican a plenitud su premio Nobel el año 1910. Los conceptos moleculares no eran rigurosos y su ecuación tenía deficiencias ya reconocidas por el mismo, pero llegaron en un tiempo crucial del desarrollo científico. Van der Waals mostró que las moléculas existían, que eran las mismas en las fases liquida y gas pero que se comportaban en forma diferente debido a fuerzas de interacción. Además mostró que el tamaño de las moléculas era importante. Se sabe también desde hace tiempo que las ecuaciones cúbicas tipo van der Waals no pueden dar resultados exactos para todas las propiedades volumétricas, termodinámicas y de equilibrio entre fases para todo tipo de fluidos y sus mezclas. De hecho, el mismo van der Waals presentaba esas dudas en su Conferencia Nobel. Por lo tanto no podemos esperar desarrollos mayores en el tema de las ecuaciones de estado hasta que conozcamos mejor como interactúan las moléculas. Sin embargo, considerando sus tremendas ventajas las ecuaciones cúbicas tipo van der Waals pueden ser ajustadas en diferentes formas para encontrar resultados aceptables en prácticamente toda situación de interés. Mi impresión es entonces que las ecuaciones cúbicas de estado derivadas de las propuestas de van Waals expuestas en su Tesis, llegaron para quedarse por un largo tiempo, o como lo expresé en mi revisión sobre ecuaciones de estado del año 2003, "cubic equations of state are here to state" (Valderrama, 2003).

$Y$ al igual que a otros pioneros de la ciencia, para van der Waals tampoco fue fácil imponer sus ideas y conceptos, pero los defendió con convicción y vehemencia desde un comienzo, con la certeza de que estaba cambiando la historia. Al final de su tesis resumía su trabajo y lo proyectaba sin falsa modestia: "He tratado de encontrar un camino a través de este campo desconocido; en algunas partes no tiene la firmeza de un camino sólido, en otros puede ser un angosto sendero, e incluso no pude evitar dar algún salto ocasional; pero aun así he traído los problemas ante los ojos de los físicos. Ya que estábamos, hasta recientemente en total ignorancia, cada sugerencia es útil, aunque dejemos algunas ideas imperfectas en lugar de una teoría perfeccionada, como vimos en el ejemplo del trabajo de James Thomson, Las dos constantes que aparecen en la teoría tienen un significado físico real, específicamente aquel del volumen y atracción molecular, y nadie puede negar que esta teoría influenciará el avance futuro en este campo".

En lo que sigue se transcribe en forma integra, y con la debida autorización de la Fundación Nobel, la conferencia que presentó van der Waals cuando recibió el merecido galardón el año 1910. Este documento forma parte también de la reciente monografía del autor, que se muestra en la Figura 2, publicada por la Editorial de la Universidad de La Serena en Chile. Después de la Conferencia se presenta una discusión final resaltando el trabajo y legado de este extraordinario científico holandés, legado que perdura hasta nuestros días. 


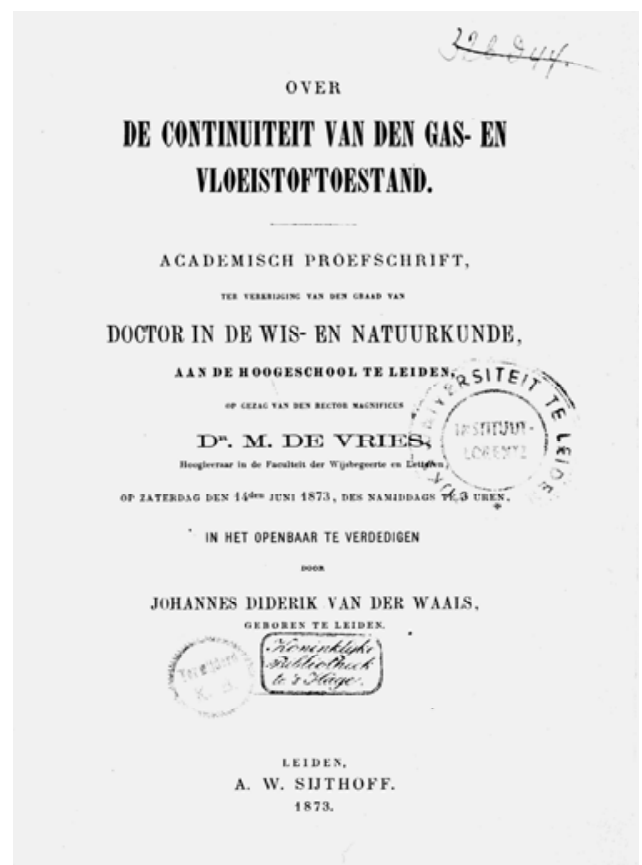

Fig. 1: Reproducción de la portadilla original de la Tesis de van der Waals (Leiden, 1873)

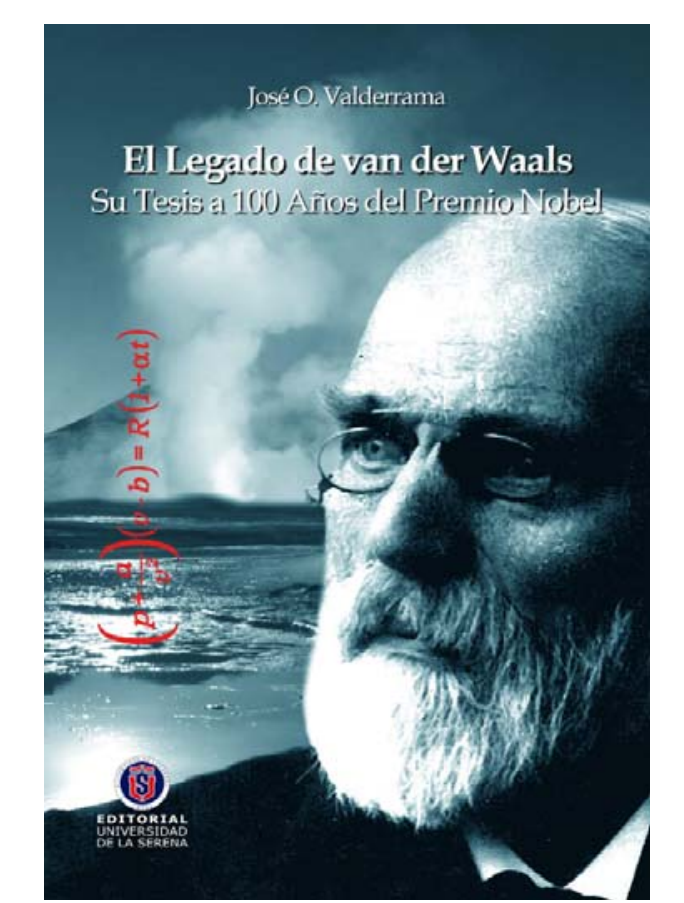

Fig. 2: Portada de la reciente monografía del autor que incluye la Tesis de van der Waals.

\section{LA CONFERENCIA NOBEL}

Ahora que tengo el privilegio de estar ante esta distinguida audiencia para hablar sobre mis estudios teóricos sobre la naturaleza de los gases y los líquidos, debo vencer la dificultad de hablar de mi mismo y de mi trabajo. Aunque la idea de que ustedes tienen derecho a esperar eso de mi, me da la esperanza de que sabrán perdonarme por presentarles mis ideas con extrema convicción, aun en asuntos que no son universalmente conocidos y que no han alcanzado reconocimiento universal. Pretendo discutir estos aspectos de manera secuencial. Parte 1: los aspectos generales de mi ecuación de estado y como llegué a ella; Parte 2: cual ha sido y es todavía mi actitud hacia esa ecuación de estado; Parte 3: como he buscado durante los últimos cuatro años la forma de considerar las diferencias entre los resultados experimentales y la ecuación; y Parte 4: como he buscado explicar el comportamiento de mezclas binarias y ternarias usando la ecuación de estado.

\section{Parte 1: Los aspectos generales de la ecuación de estado}

Los primeros incentivos en mi trabajo de vida llegaron cuando, después de mis estudios universitarios, leí el tratado de Clausius (1857) sobre la naturaleza del movimiento que nosotros llamamos calor. En ese tratado, que ahora nosotros enseñamos con muy pequeñas modificaciones en todas las escuelas superiores de Holanda, el mostró como la ley de Boyle puede ser realmente derivada suponiendo que un gas consiste de puntos materiales que se mueven a gran velocidad, y que esta velocidad es del orden de la del sonido y que aumenta en proporción a la raíz cuadrada de la temperatura absoluta. Luego se descubrió que solo era la raíz cuadrática media de las velocidades y que, como Maxwell lo estableció, aunque el erróneamente consideró que lo había demostrado, que hay una ley para la distribución de velocidades conocida hoy como ley de Maxwell. Boltzman fue el primero en dar una prueba correcta para la ley de distribución de velocidades.

El tratado de Clausius fue una revelación para mi aunque pensaba al mismo tiempo que un gas en un estado extremadamente diluido, en el que el volumen es tan grande que las moléculas pueden ser consideradas como puntos, consiste en pequeñas partículas que se mueven, lo que es obviamente válido cuando el volumen es pequeño; en realidad tal debe ser el caso hasta la máxima compresión y también para los líquidos, los que pueden ser solamente considerados como gases a baja temperatura. Por lo tanto concebí la idea de que no hay diferencia esencial entre el estado líquido y el gaseoso de la materia, y que los factores, aparte del movimiento de las moléculas, que actúan para determinar 
la presión deben ser considerados como cuantitativamente diferentes cuando cambia la densidad y talvez también cuando cambia la temperatura, pero esos deben ser los mismos factores que influencian el sistema. $\mathrm{Y}$ entonces se me ocurrió la idea de continuidad. Y me pregunté también como se comportaría el estado sólido. $Y$ aunque no trabajé seriamente en responder esa pregunta pienso que en un estado amorfo la proximidad cercana de las moléculas impiden su mutuo desplazamiento. El estado cristalino se comporta de una manera diferente. En realidad debería quedarme callado frente a esta cuestión. Sin embargo es raro hoy en día dudar de que exista continuidad entre los otros dos estados de agregación.

Como ustedes saben, los dos factores que yo he especificado como las razones para que un agregado diluido de partículas en movimiento no cumpla con la ley de Boyle son, primero la atracción entre las partículas y segundo su propio volumen. Con respecto al primer factor me gustaría puntualizar lo siguiente: originalmente yo había anticipado que simplemente al volumen total se le debe restar el volumen de las moléculas para encontrar el volumen remanente donde las partículas se pueden mover. Pero un examen más detallado me indicó que el asunto no era tan simple. Para mi sorpresa, me di cuenta de que la magnitud en la que disminuía el volumen era variable, que en el extremo del estado muy diluido esta cantidad, que yo he denotado por $b$ es cuatro veces el volumen molecular, pero que esta cantidad decrece a medida que el volumen extremo decrece y gradualmente cae hasta la mitad. Pero las leyes que gobiernan este decrecimiento no han sido aún encontradas. Este punto de vista ha probado ser el más difícil de estudiar en la ecuación de estado. Korteweg, Lorentz, Boltzmann, Jeans, y mis estudiantes, Van Laar, mi hijo, y Kohnstamm han trabajado en ello.

Yo había pensado que era simplemente estudiar como disminuía el camino libre medio entre las colisiones de las moléculas que es la consecuencia de su extensión, es decir el hecho de que ellas no deban ser consideradas puntos materiales sino pequeñas partículas con un volumen real similar a cualquier cuerpo conocido por nosotros, y entonces llegué a esta fórmula:

$b=b_{g}\left[1-\alpha \frac{b_{g}}{v}+\beta\left(\frac{b_{g}}{v}\right)^{2}\right.$ etc. $]$

donde $b_{g}$ es cuatro veces el volumen molecular.

Boltzmann ha mostrado sin embargo, que esto es inadecuado y más tarde Kohnstamm demostró que la fórmula sería más compleja y que para $b / b_{g}$ se obtendría un cuociente de dos series en las que aparecen potencias de $b_{g} / v$. Como es difícil determinar los coeficientes $a, b$ y los otros, van Laar debió realizar largos y tediosos cálculos para determinar el segundo coeficiente $b$ por mi método. Esto evitó que yo continuara en esa línea. Y llegué al punto débil de la ecuación de estado. Yo aún me pregunto si hay o no otra forma mejor. En realidad yo estaba obsesionado por la idea de continuidad y nunca pude apartarme de ella, y estaba incluso en mis sueños. Con respecto a la otra causa por la que los gases reales y los líquidos no cumplían la ley de Boyle (1660), me refiero a la atracción entre las moléculas, la situación es un poco mejor aunque aun no se ha dicho la última palabra. Usando el procedimiento adoptado por Laplace en su teoría de la capilaridad, yo había reducido esta atracción, que actúa sobre todo el volumen, a una fuerza de superficie que actúa hacia el interior y que junto con la presión externa mantiene juntas a las moléculas en movimiento.

Laplace consideraba su líquido como un medio continuo, pero al mismo tiempo él no sabia de la existencia de las moléculas. Y si nosotros estuviéramos tratando con moléculas en estado estacionario sería inadmisible reducir las fuerzas atractivas del interior solo a fuerzas de superficie. Y como las moléculas están en movimiento, cada punto en el interior del sistema no estará lleno de materia en todo momento. Pero el espacio puede ser considerado como lleno en forma continua por materia de densidad media-normal. Yo regresaré a este punto cuando hable de mis estudios en los años más recientes. Las consideraciones indicadas más arriba me llevaron a la siguiente fórmula:

$P=\frac{R T}{v-b}-\frac{a}{v^{2}}$

que ha llegado a ser universalmente conocida gracias a los esfuerzos de Eilhard Wiedemann. 


\section{Parte 2: Mi actitud hacia la ecuación de estado}

Habiendo llegado a la segunda parte de mi conferencia debo mencionar ahora mi propia actitud hacia esta ecuación. Seré muy claro en indicar que a partir de mis comentarios previos yo nunca esperé que esta ecuación con $a$ y $b$ constantes diera resultados numéricos que concordaran con los experimentales y aun así la gente actúa siempre como si esa fuera mi opinión. Esto me sorprende por cierto ya que en mi Tesis de 1873 no solo expresé la variabilidad de $b$ sino que además presenté algunos valores de $b$ a partir de los experimentos de Andrews, en los que calculé los cambios en $b$ para pequeños volúmenes. Para dióxido de carbono yo di valores de $b_{g}=0.0023$ hasta $b=0.001565$. En esta serie de valores, los volúmenes del líquido incluso son más bajos que el valor de $b_{g}$ para volumen infinito. Talvez la razón por la cual mi opinión de que $b$ es variable ha sido raramente considerada, debe ser en la manera que yo calculé los parámetros críticos. En estos cálculos debí suponer que $b$ era invariable. Pero esto fue porque yo creí que los valores de Andrews se justificaban aunque yo le asignara el valor límite $b_{g}$ a $b$ en el punto crítico. Pero como mostré más tarde eso era un error; el valor de $b$ en el punto critico tiene que ser, por cierto, ligeramente disminuido. Así para determinar el volumen critico cuando $b$ cambia con el volumen, la primera y segunda derivada $d b / d v$ y $d^{2} b / d v^{2}$ también necesitan ser conocidas. Para determinar el volumen critico, como yo mostré más tarde, obtenemos la siguiente ecuación:

$$
\frac{v}{v-b}(1-d b / d t)+\frac{1}{2}\left[\frac{v\left(d^{2} b / d v^{2}\right)}{1-d b / d v)}\right]=\frac{3}{2}
$$

Solamente cuando despreciamos $d b / d v$ y $d^{2} b / d v^{2}$ encontramos que $v_{k}=3 b$. Y puede verse de nuevo de esta ecuación que lo que he denominado el punto débil de mi teoría es en realidad el responsable de la imposibilidad teórica de calcular en forma exacta el volumen crítico. Usando una fórmula aproximada para b pude determinar que $v_{k}$ es aproximadamente igual a $2.2 b_{g}$. Yo usé esta ecuación para determinar el volumen crítico en mi trabajo en honor a Boltzmann y la repetí en parte en mi trabajo de 1910. Cuando me di cuenta de esta gran diferencia en el cálculo del volumen crítico yo también temí que habría gran diferencia en las otras propiedades críticas, es decir que no seguirían las fórmulas: $R T_{k}=8 a / 27 b_{g}$ y $p_{k}=1 / 27 b_{g}{ }^{2}$

Para mi gran satisfacción, sin embargo, y no exento de sorpresa, encontré que estas dos ecuaciones usadas para calcular $a$ y $b$ a partir de los datos críticos permanecían sin cambio. Eso lo pude concluir de los resultados de los admirables experimentos sobre volúmenes y coexistencia de la fase líquida y vapor y sobre la presión de saturación a varias temperaturas realizadas por Sydney Young. Yo presenté recientemente estos resultados a los que me referiré de nuevo en esta conferencia. Sin embargo puedo explicar esto como sigue. Sydney Young determinó, entre otros asuntos, el valor de

en el punto critico y encontró 1/3.77. Si $p$ y $T$ tienen el mismo valor que en mi teoría, manteniendo b constante, la diferencia con mi valor de $3 / 8$, que es el valor que yo encuentro de $p v / R T$, puede ser atribuida completamente al volumen. $Y$ esto, podemos decir, es completamente correcto. El volumen critico no es $3 b_{g}$ sino $(3 / h) b_{g}=2.125 b_{g}$ (aproximadamente). $Y$ el término $p v / R T$ no es $3 / 8$ sino $3 / 8 \sqrt{ } 2$.

Para el valor de

$$
\left(\frac{T}{p} \frac{d p}{d T}\right)_{k}-1
$$

mi ecuación da $a / p v_{k}^{2}$. Usando los datos experimentales de Sydney Young se encuentra un valor de alrededor de 6 para el termino de arriba. Por lo tanto para $p_{k}$ tenemos el valor $p_{k}=a / 6 v_{k}^{2}$ y con $v_{k}=$ $\left(3 b_{g} / \sqrt{2}\right)$ se obtiene para $p_{k}$ el valor original $\left.p_{k}=(1 / 27) a / b_{g}^{2}\right)$. Se verá por lo tanto que yo nunca he considerado que se ha dicho la última palabra acerca de la ecuación de estado y yo he regresado 
continuamente a ella durante mis estudios. Ya en el año 1873 yo reconocía la posibilidad de que $a$ y $b$ pudieran variar con la temperatura, y es bien sabido que incluso Clausius supuso que a variaba en forma inversamente proporcional a la temperatura absoluta. Entonces Clausius pensó que si consideraba esta ecuación

$\left(\frac{T}{p} \frac{d p}{d T}-1\right)_{k}=6$

el resultado es solo la mitad cuando $a$ y $b$ se mantienen constantes.

Los conceptos anteriores significan que este valor no proviene de un cambio con la temperatura sino simplemente de un cambio de $b$ con el volumen. Por mucho tiempo yo he tratado de encontrar una característica definida para determinar si solamente haciendo $b$ variable sería suficiente para que los valores calculados con mi fórmula y los datos experimentales concuerden; y en el caso de haber discrepancias ver si es necesario talvez suponer variabilidad de $a$ y de $b$ con la temperatura. Dicho esto, paso a describir mis últimos estudios sobre la ecuación de estado.

\section{Parte 3: Diferencias entre los resultados experimentales y la ecuación}

En el año 1906 ofrecí una conferencia en la Real Academia de Ciencias de Amsterdam, titulada "seudo-asociación". En ese tiempo, sin embargo, me conformé con una exposición oral. Pero uno de mis discípulos, el Dr. Hallo, hizo notas escritas sobre ello y más tarde el Dr. Van Rij lo incorporó en una disertación inaugural y desarrollo más el tema. Como criterio para determinar si la variabilidad de $b$ con el volumen sería suficiente para encontrar acuerdo entre los resultados de mi fórmula y los volúmenes experimentales del líquido y vapor determinados por Sydney Young, yo usé en mi conferencia el principio de Clapeyron el cual, con $b$ variando solo con $v$ y no con $T$, conduce a la ecuación

$$
\left(\frac{T}{p} \frac{d p}{d T}-1\right) p=\frac{a / v_{1}-a / v_{2}}{v_{1}-v_{2}}=\frac{a}{v_{1} v_{2}}
$$

o bien

$$
\left(\frac{T}{p} \frac{d p}{d T}-1\right) p v_{1} v_{2}=a
$$

Para asegurar que solamente hay parámetros experimentalmente determinables podemos escribir también

$$
\left(\frac{T}{p} \frac{d p}{d T}-1\right) p v_{1} v_{2} /\left(\frac{T}{p} \frac{d p}{d T}-1\right)=1
$$

Todos los parámetros a la izquierda de la ecuación fueron determinados con exactitud por Sydney Young para una serie de sustancias. A la temperatura crítica el valor del lado izquierdo de la ecuación es por cierto igual a la unidad; ¿pero cual es su valor a una temperatura que sea solo una fracción de la crítica $T_{k}$ ?. Aquí, al parecer, a medida que la temperatura disminuye el valor en el lado izquierdo aumenta muy rápidamente al comienzo y después a una velocidad imperceptible. Los experimentos de Sydney Young fueron solo hasta valores de $T / T_{k}=2 / 3$. El lado derecho de la ecuación (5) ha aumentado hasta 1.4 y parece aproximarse asintóticamente al valor 1.5. El valor del lado derecho puede ser bien representado por la fórmula empírica, con $T / T_{k}=m$ :

$$
a+\sqrt{1-m}-\frac{1-m}{2}
$$

A temperaturas muy cercanas a la critica, por ej. $m=0.99$, el aumento en esta expresión llega a ser 0.1 , mientras que si la raíz cuadrada de $(1-m)$ no es incluida en la fórmula, y el aumento solo estuviera representado por $(1-m)$, este sería menor por un factor de diez. 
Después debí examinar si la suposición de que si a o $b$ eran dependientes de la temperatura, ello podría representar correctamente el aumento del lado derecho de la ecuación (5); y si ese no era el caso ver si otra expresión para la presión interna, que yo siempre escribí como $a / V^{2}$ podría explicarlo. Como resultado de este análisis se encontró que las funciones de la temperatura para $a$ y $b$, concordante con el rápido aumento inicial del lado derecho, debía contener el término $\sqrt{ }(1-m)$. Por sobre la temperatura crítica, sin embargo, esto daría valores imaginarios para a y $b$, lo que queda fuera de lugar considerando el comportamiento general de los gases. Al parecer, tampoco hay otra expresión para la presión interna que pueda conducir a un rápido aumento inicial como el que se discute. No se debe olvidar que la temperatura crítica no es una temperatura especial. A esta temperatura las densidades coexistentes son iguales en magnitud. Eso es lo único que le da a esta temperatura un significado especial que bajo toda otra circunstancia no tiene. Si ocurriera un salto brusco o sea un rápido incremento ya sea de $a$ o de $b$ haría que esa temperatura fuera bastante especial a cualquier nivel de compresión y su determinación sería entonces posible cualquiera fuera la densidad.

Hablando en forma simple, el resultado sería que es imposible encontrar una ecuación de estado compatible con los datos experimentales. En efecto, no existe tal ecuación de estado, a menos que algo sea agregado; por ejemplo que las moléculas se asocian para formar complejos más grandes. Este año he escrito dos artículos para la Academia de Amsterdam sobre esta posible asociación. La he denominado "seudo-asociación", para diferenciarla de la asociación que es de origen químico. La posible formación de complejos moleculares, particularmente en el estado líquido ha sido frecuentemente enfatizada, y el hecho de que tal suposición es necesaria para lograr concordancia entre la ecuación de estado y los experimentos no debería causar sorpresa. Desafortunadamente, mi examen no es aún completo. Ha sido difícil, y he debido hacer uso de cada evidencia para llegar a algo concreto. Sin embargo, se sabe hoy lo suficiente como para suponer que un gran número de moléculas solas son necesarias para formar un nuevo grupo que las retenga juntas y se comporte como una nueva unidad grande en movimiento molecular.

¿Cual es el origen de esta formación de complejos, de esta seudo asociación?. Yo me he visto obligado a suponerla porque parece ser que es la única forma de construir una ecuación de estado, cualquiera que ella sea, compatible con los resultados experimentales. Sin embargo, como resultado de una observación de Debye en la edición del mes pasado de la revista "Sobre la Física" (An den der Physik), yo recordé una frase de Boltzmann. Hace unos años, cuando tuve el privilegio de tenerlo unos días en mi laboratorio, entre las muchas cuestiones que discutimos, me dijo que no podía explicar la atracción de las moléculas solo mediante una fuerza superficial. En ese momento, la frase de Boltzmann no me dijo mucho, y solo ahora veo lo que significaba. Volviendo a mi Tesis del año 1873, yo llegué a la conclusión de que la atracción de las moléculas disminuía muy rápidamente con la distancia, y que la atracción era apreciable solamente a distancias cercanas al tamaño de las moléculas. En ese tiempo me atreví incluso a decir que en el caso de los gases solamente las colisiones eran las responsables por las fuerzas de atracción. Y adopté la visión de Boltzmann, de que era solo posible admitir el procedimiento de Laplace y suponer una fuerza de superficie como la consecuencia de la atracción, provisto de que no caiga tan rápido (Boltzmann, 1898). Lo establecido por Debye implica que Boltzmann había predicho la formación de un complejo.

Por lo tanto, creo yo, la suposición de seudo asociación se justifica desde el punto de vista teórico. Y ahora creo que puedo relatar como he procedido en mi último trabajo que apareció en noviembre de este año. La seudo asociación difiere de la verdadera asociación en que esta última es el resultado de nuevas fuerzas químicas que solo aparecen cuando las moléculas están combinadas; por ejemplo formando dobles moléculas, mientras que la seudo asociación puede ser atribuida solamente a fuerzas moleculares normales. Ahora bien, ya que estas fuerzas disminuyen tan rápido, hay dos consecuencias. Primero resulta en la formación de un complejo y segundo conduce a una presión de superficie, aunque a una menor. $Y$ es precisamente esto lo que debí suponer en mis cálculos y que se explica de la siguiente manera. Supongamos que el número de moléculas que se han combinado en el complejo es tan grande que es posible hablar de una molécula central rodeada por una capa conteniendo tantas moléculas como sea posible al mismo tiempo. Entonces, para las moléculas de la capa, la atracción dirigida hacia el interior actúa solo para mantener el complejo, y parte de su atracción se pierde por la presión de superficie. Solo las fuerzas que actúan hacia afuera de estas moléculas pueden contribuir a la formación de la presión interna. Pero por cierto, tanto para la asociación 
como para la seudo asociación el número de complejos formados aumenta con la disminución de la temperatura y el volumen. Por lo tanto eso me llevó a concluir que en el punto crítico solo una pequeña parte de la masa está presente formando complejos. Si la seudo asociación existe en una sustancia, hay al menos dos tipos de moléculas: simples y complejas. Digo al menos dos tipos, porque no se puede suponer en una primera etapa que todos los complejos son de igual tamaño. Pero en una primera etapa he supuesto solo dos tipos, esto es moléculas simples y complejos n-moléculas. Para un tratamiento realmente científico, por cierto, sería necesario suponer todos los valores de $n$ como posibles y buscar una ley de distribución para estos valores. Por ahora sin embargo, me concentraré solo en un tipo de complejo. Tenemos entonces una mezcla binaria. Fue muy afortunado que por muchos años estudiara las leyes de las mezclas binarias; y así he llegado al cuarto punto de mi conferencia. Para no ocupar mucho de vuestro tiempo sin embargo, prometo ser breve.

\section{Parte 4: Comportamiento de mezclas usando la ecuación de estado}

No puedo decir exactamente cuando concebí la idea de utilizar mi ecuación para estudiar las propiedades de mezclas binarias. Pero hace al menos 20 años a instancias de mi amigo Kamerlingh-Onnes, publiqué una completa teoría sobre mezclas binarias. Mi "Théorie moléculaire d'une substance composée de deux matières différentes" (Teoría molecular de una sustancia compuesta de dos materias diferentes), apareció en los Archivos Holandeses el año 1890 (van der Waals, 1890). Fue escrito en Holandés pero mí estimado amigo Bosscha se tomó la ardua tarea de ponerlo en Francés, una tarea que fue aun más excitante ya que yo la había escrito en una forma muy concisa y el tratamiento matemático conducía a puntos trenzas (plait points) y a curvas particulares que hasta ese tiempo habían sido raramente analizadas con detalle. Previamente, sin embargo, mi amigo Korteweg, con quien había comentado en términos generales las conclusiones de mis análisis, había estudiado las propiedades matemáticas de estos puntos y curvas, un estudio que he encontrado de gran utilidad. Las razones por las que dudé un largo tiempo en publicarlas fueron muchas, y son de poca utilidad discutirlas aquí.

Pero una de las razones de importancia científica fue la pregunta que me hacía con frecuencia: ¿Sirve de algo aplicar la ecuación de estado a mezclas sin haberla estudiado en forma completa para sustancias puras? Yo postulaba que mientras no se conocieran las leyes que gobiernan la variabilidad de $b$ y tuviera que suponer que $b$ no variaba con el volumen, los resultados para muchos parámetros conducirían a valores muy diferentes a los experimentales. Sin embargo, la consideración que aun con $b$ constante mi teoría era importante para el caso de una sustancia pura me dio la esperanza de que muchos fenómenos serían cualitativamente explicados si se introducían valores apropiados de $a$ y $b$ para las mezclas. Fue de gran importancia para mi conocer los tratados de Gibbs sobre equilibrio de sustancias heterogéneas que el me envió en cuanto las publicó. Entre otras cosas hice uso de su teoría de que una cantidad de sustancia alcanza el equilibrio si la energía libre es mínima para una temperatura y volumen dados. En su honor yo he denominado la superficie de equilibrio para un sistema binario como superficie- $\psi$. Esto porque Gibbs siempre representó con el signo $\psi$ a la energía libre, cuyo significado para el equilibrio fue el primero en reconocer.

Los fenómenos no son complejos en el caso de una sustancia simple y se puede obtener un cuadro general en forma fácil. Es por lo tanto muy sorprendente que en una simple mezcla binaria ellos sean tan complejos que han llegado a ser considerados con frecuencia como un laberinto. Esto es particularmente cierto en situaciones en la que puede existir la presión de tres fases. Y ahora es claro que los fenómenos son al menos cualitativamente concordantes con la "teoría molecular de una sustancia compuesta de dos materias diferentes", a partir de la cual dichos fenómenos pueden ser derivados y en realidad predichos. Debido a los muchos estudios experimentales a los que dicha teoría ha dado origen he tenido la oportunidad de discutirla en varias ocasiones. Entre los años 1907 y 1909 aparecieron cerca de 15 publicaciones sobre el tema en los anales de la Academia de Amsterdam. Sería largo mencionar los nombres de los físicos y químicos que, siguiendo mi teoría, han estudiado y continúan estudiando las mezclas binarias. Empieza con Kuenen y termina con el Dr. Jean Timmermans de Bélgica cuyos estudios aún continúan y por ello han sido solo parcialmente publicados.

Ahora si me permiten retroceder a sobre la forma en que llegué a ella, debo confesar que para ser justo debería llamarla un desvío. Tan pronto como la necesidad de suponer asociación fue clara para 
mi yo extendí la ecuación de estado en forma análoga a la fórmula para la mezcla binaria e introduje un nuevo parámetro, el grado de asociación. Este grado de asociación es determinado por medio de la ecuación de Gibbs a la que me he referido. El grado de asociación así determinado debe ser introducido en la ecuación de estado. Y debo confesar que este es un desvío. Talvez haya una manera directa y que está siendo seriamente investigada por aquellos que tienen contacto directo conmigo. En la búsqueda de esta forma directa, la obra Principios Elementales de Mecánica Estadística de Gibbs (1902) será una guía necesaria. En esta conferencia yo solo he contado la historia del origen y posterior elaboración de mis estudios teóricos y por lo tanto tenía que hablar de las dificultades que debieron superarse. Consecuentemente, yo no podría, o solo escasamente discutir como dichos principios elementales han ayudado a comprender correctamente los fenómenos.

Una de las principales conclusiones que he denominado "Principio de los Estados Correspondientes", ha llegado a ser universalmente conocida (van der Waals, 1880). Yo no he discutido como este principio ha sido un factor determinante en el método de Dewar para la producción de hidrogeno liquido, y particularmente en la determinación del método de licuefacción de helio de Kamerlingh-Onnes. Tampoco he dado una detallada discusión de la temperatura a la cual, para usar la nomenclatura de Regnault, un gas empieza a comportarse como un "gas más que perfecto" (gaz plus que parfait). Se ha encontrado que dicha temperatura debe ser (27/8) $T_{k}$, y además la temperatura a la que se puede decir que el efecto Joule-Kelvin se invierte debe ser (27/4) $T_{k}$.

Tampoco he hablado de mi talvez excesivo esfuerzo para determinar la ecuación de estado de la molécula misma. He incluso lo evitado decir, porque pensaba que debía determinar primero la relación entre $p, v$ y $T$. Las fórmulas de la termodinámica son efectivas y pueden ser realmente aplicadas a todos los problemas, aun para determinar los parámetros térmicos, solo cuando esta relación entre $p$, $\checkmark$ y $T$ es conocida, o de lo contrario solo puede ser considerada como una ecuación con dos incógnitas. Yo he enfatizado explícitamente esto en el libro publicado en conmemoración de KamerlinghOnnes publicado en 1904. Pero no ha sido mi intención discutir todo esto y no tendría el tiempo suficiente. Aun así no me parece superfluo, y talvez es incluso necesario hacer una observación general. Debe quedar perfectamente claro que en todos mis estudios yo estaba muy convencido de la real existencia de las moléculas, que nunca las consideré como un invento de mi imaginación, ni siquiera como centros de los efectos de fuerza. Yo las consideraba como cuerpos reales, de modo que el término "cuerpo" en lenguaje diario debería ser llamado "seudo cuerpo". Se trata de un agregado de cuerpos y espacio vacío. Nosotros no sabemos aún la naturaleza de una molécula que consiste de un solo átomo químico.

Sería muy prematuro buscar una respuesta a esta pregunta, pero admitir esta ignorancia no perjudica la creencia en su verdadera existencia. Cuando empecé mis estudios sentía que yo era el único que tenia esta visión. Y cuando, como ya ocurrió con mi Tesis de 1873, yo determiné el número de moléculas en un gramo-mol, su tamaño y la naturaleza de sus acciones, yo había reforzado mi opinión; aunque a veces me pregunto si en el análisis final una molécula es un invento de mi imaginación y la teoría molecular completa también. Y ahora yo no creo que sea una exageración establecer que la real existencia de las moléculas es universalmente supuesta por los físicos. Muchos de aquellos que se oponían han sido finalmente derrotados y mi teoría puede haber sido un factor determinante. $Y$ precisamente esto, creo, es un avance. Cualquiera que esté familiarizado con los escritos de Boltzmann y de Willard Gibbs admitirán que los físicos de gran renombre creen que los complejos fenómenos de la teoría del calor pueden ser interpretados solamente de esta forma. Es un gran placer para mí constatar que un número creciente de jóvenes físicos encuentran inspiración en mis trabajos y visión sobre la teoría molecular. La coronación de mis estudios por la muy estimada Real Academia Sueca me llena de satisfacción y compromete mi gratitud, gratitud que no puede ser eterna y a mi avanzada edad no puedo prometer que será de larga duración, pero talvez por esta razón mi gratitud es aún más intensa.

\section{DISCUSIÓN FINAL}

Los conceptos relacionados con tamaño y atracción entre las moléculas, el principio de estados correspondientes, y la teoría de mezclas binarias son las contribuciones más notables de van der Waals. Pero van der Waals no solo contribuyó en estas áreas. La suposición de campo-medio (mean-field assumption) es una extraordinaria contribución para el desarrollo de la mecánica estadística; y su teoría de la 
capilaridad, que aunque menos mencionada, es reconocida como otro de sus grandes aportes. Sin embargo, la habilidad de su ecuación de estado para describir una serie de fenómenos aún desconocidos en su época y la serie de teorías deducidas a partir de ella, son sin duda las contribuciones más destacables de su trabajo científico y son materias de estudio hasta hoy en día. No hay prácticamente ningún desarrollo actual en el área de la física de fluidos que no considere los aspectos básicos de las teorías de van der Waals. Por ello es que su legado ha trascendido el tiempo, las fronteras geográficas y las diferencias de idioma; y este año estamos justo a un siglo desde que su extraordinario trabajo fuera reconocido por la Academia Sueca otorgándole el máximo galardón en Física el año 1910. El libro de Levelt-Sengers [2002] "How Fluids Unmix", escrito para celebrar los 250 años de la Real Sociedad Holandesa de Ciencias y Humanidades, hace un detallado recuento y un reconocimiento explicito de las notables contribuciones de van der Waals y de la ciencia holandesa al desarrollo del conocimiento.

Probablemente van der Waals no sospechaba que uno de los aspectos no centrales de su tema de Tesis provocaría un cambio tan notable en el conocimiento sobre el comportamiento de fluidos y haría trascender su trabajo por generaciones. Es conveniente resaltar aquí la primera frase del Prefacio de su celebrada Tesis para ver que en un comienzo sus intereses estaban más en entender un aspecto distinto del comportamiento de los líquidos: "La elección del tema que constituye el material para el presente tratado surgió por el deseo de entender una magnitud que juega un rol especial en la teoría de la Capilaridad desarrollada por Laplace. Es la magnitud que representa la presión molecular ejercida por un líquido, rodeado por una superficie plana, sobre una unidad de esta superficie.". Y, si bien van der Waals realizó otros importantes aportes, como se menciona más arriba, son los conceptos sobre la existencia y extensión de las moléculas y sobre interacción molecular, que se plasman en forma magistral en la ecuación de estado y otras teorías derivadas de ella, lo más trascendental de su obra. En efecto, ese es el motivo principal de su premio Nobel, como se lee en la presentación de tan alta distinción que realizara el entonces Presidente de la Real Academia de Ciencias de Suecia, Profesor Oscar Montelius, el 12 de Diciembre de 1910: "Profesor Van der Waals, la Real Academia de Ciencias ha otorgado a Ud. este año el Premio Nobel de Física en reconocimiento a sus pioneros estudios sobre la física de líquidos y gases" (Nobel.org, 2010b). Y por ser lo más notable y trascendente de su obra es que la califico, sin duda alguna, como su mayor y más importante legado.

\section{REFERENCIAS}

Andrews, T., The Bakerian Lecture: On the Continuity of the Gaseous and Liquid States of Matter, Phil. Trans. Roy. Soc., 159, 575-590 (1869)

Boltzmann, L. (1898): Vorlesungen über Gastheorie. II Teil. Leipzig, J. A. Barth (1898).

Boyle, R., New Experiments Physico-Mechanical. Touching the Spring of the Air, Oxford (1660)

Clausius, R., Ann. Phys. Over the Behavior of the Carbon Dioxide with regard to Pressure, Volume and Temperature, 9, 337-357 (1880)

Gibbs, J.W., Elementary Principles of Statistical Mechanics, Edward Anold, London (1902)

Levelt-Sengers, J., How Fluids Unmix. Discoveries by the School of Van der Waals and KamerlinghOnnes, Royal Netherlands Academy of Arts and Sciences, Amsterdam-Holland (2002)

Nobel.org, http://nobelprize.org/nobel_prizes/physics/laureates/1910 (2010a)

Nobel.org, http://nobelprize.org/nobel_prizes/physics/laureates/1910/press.html (2010b)

Valderrama, J.O. The State of the Cubic Equations of State, Ind. Eng. Chem. Res. 42 (7) 1603-1618 (2003)

Valderrama, J.O. The Legacy of van der Waals. His Thesis after 1000 Years Since the Nobel Prize, Edit. University of La Serena, La Serena-Chile (2010)

van der Waals, J.D., On the Continuity of the Gaseous and Liquid State, Dissertation Universidad de Leiden, Holanda (1873)

van der Waals, J.D., Investigations on the Corresponding Properties of the Normal Saturated Vapor and Liquid Curves for Different Fluids, Verhand. Kon. Akad. 20(5), 1-32 (1880)

van der Waals, J.D., Molecular theory of a Substance composed of Two Different Species], Z. Physik. Chem. 5, 173-193 (1890)

van der Waals, J.D. The equation of state for Gases and Liquids, Nobel lecture (1910) 